\title{
Regulatory T, natural killer T and $\gamma \delta$ T cells in multiple sclerosis and chronic fatigue syndrome/myalgic encephalomyelitis: a comparison
}

Sandra Ramos, ${ }^{1,2}$ Ekua Brenu, ${ }^{1,2}$ Simon Broadley, ${ }^{1,2}$ Richard Kwiatek, ${ }^{3}$ Jennifer $\mathrm{Ng}^{2,4}$ Thao Nguyen, ${ }^{1,2}$ Susan Freeman,${ }^{2}$ Donald Staines, ${ }^{2}$ Sonya Marshall-Gradisnik ${ }^{1,2}$

\begin{abstract}
Background: Chronic Fatigue Syndrome/Myalgic Encephalomyelitis (CFS/ME), and Multiple Sclerosis (MS) may share some similarities in relation to reduced NK cell activity. It is likely that other cells such as regulatory T (Tregs), invariant Natural Killer T (iNKT) and gamma delta T $(\gamma \delta \mathrm{T})$ cells may also be dysregulated in CFS/ME and MS.

Objective: To evaluate and compare specific immune regulatory cells of patients with CFS/ME, patients with MS and healthy controls.

Method: Sixty three volunteers were included in this study: 24 were CFS/ME patients, 11 were MS patients and 27 were healthy controls. Blood samples were obtained from all participants for flow cytometry analysis of iNKT cells, Tregs and $\gamma \delta \mathrm{T}$ cell phenotypes.

Results: We observed a significant increase in Tregs in the CFS/ME group $(\mathrm{p} \leq 0.05)$ compared to the healthy control group. Total $\gamma \delta$ and $\gamma \delta 2$ T cells were significantly reduced in MS patients in comparison with the healthy control group. Conversely, CD4 ${ }^{+}$iNKT percentage of iNKT, was significantly increased in the CFS/ME group compared with healthy controls and the double-negative iNKT percentage of iNKT significantly decreased compared with the healthy control group.

Conclusions: This study has not identified any immunological disturbances that are common in both MS and CFS/ME patients. However, the differential expression of cell types between the conditions investigated suggests different pathways of disease. These differences need to be explored in further studies.
\end{abstract}

Keywords: Chronic Fatigue Syndrome; Myalgic Encephalomyelitis; Multiple Sclerosis; Regulatory T cells, natural killer T cells; $\gamma \delta$ T cells.

From:

${ }^{1}$ Griffith Health Institute - Griffith University, Gold Coast, QLD, Australia

${ }^{2}$ National Centre for Neuroimmunology and Emerging Diseases (NCNED) - Griffith University, Gold Coast, QLD, Australia

${ }^{3}$ Northern Adelaide Local Health Network, Adelaide, SA, Australia

${ }^{4}$ Paradise Arthritis and Rheumatology Clinic, Gold Coast, QLD, Australia

\section{Corresponding author:}

Thao Nguyen

Gold Coast Griffith University,

Level 8, Mailbox 64 Parklands Drive, Southport, Gold Coast,

QLD, Australia, 4222

Email: thao.nguyen2@griffithuni.edu.au

\section{Introduction}

Chronic Fatigue Syndrome/Myalgic Encephalomyelitis (CFS/ME) is characterized by unexplained disabling fatigue which is regularly accompanied by flu-like symptoms such as unrefreshing sleep, impaired concentration or short-term memory, post-exertional malaise, muscle and joint pain, headaches, and sore throats that persist for over 6 months. ${ }^{1}$ Patients with CFS/ME often present with symptoms including impaired cognition, immune and endocrine dysfunction and may have a poor prognosis of recovery. ${ }^{1}$ No diagnostic test has been established for CFS/ME. Hence, a plausible biological pathophysiology remains to be determined. Multiple Sclerosis (MS) is an autoimmune inflammatory disorder of the central nervous system (CNS). The classical order of pathological events in MS includes chronic inflammation, primary demyelination, 
and axonal damage. ${ }^{2,3}$ Regardless of the vast research on MS, the pathogenic mechanisms involved in the inflammatory process of MS remain unclear.

Immune cells that have both an innate and adaptive immune response and are a bridge between the two immune systems include regulatory $\mathrm{T}$ (Treg) cells, invariant natural killer (iNKT) cells and gamma delta $(\gamma \delta) \mathrm{T}$ cells. Immune dysfunction has been shown to play a substantial role in the symptomatology of CFS/ME. Previous investigations have repeatedly reported reduced natural killer (NK) cell cytotoxicity in CFS/ME patients compared with healthy controls. ${ }^{4,5}$ Similarly, increased regulatory $\mathrm{T}$ cells (Tregs) in CFS/ME patients have been identified consistently. ${ }^{4-6}$

NK cells have previously been investigated in MS patients under therapies including daclizumab, interferon- $\beta$, natalizumab, and glatiramer acetate. ${ }^{7}$ In MS patients, these therapies have the ability to increase NK numbers and/or function. This highlights an important role of NK cells and their function in MS. ${ }^{8}$ Tregs have a key role in other presumed autoimmune diseases such as MS; however, there is no reported difference in the frequency of Tregs in MS patients in comparison to healthy individuals. ${ }^{9}$

Invariant Natural killer T (iNKT) cells and $\gamma \delta$ T cells have been examined in CFS/ME and MS patients, where iNKT cells suppress and inhibit cytokine production of autoreactive cells. $\gamma \delta$ T cells on the other hand interact with dendritic cells in the priming of the adaptive immune response and, once activated in the periphery, $\gamma \delta \mathrm{T}$ cells may confer an antigen-presenting function. ${ }^{10}$ Previous research reported a lack of circulating $\mathrm{T}$ cells expressing the NK markers CD56 ${ }^{+}$ and $\mathrm{CD}_{161^{+}}$and $\mathrm{T}$ cell receptor (TCR) in patients with MS. ${ }^{11}$ Moreover, the iNKT cells from MS subjects in the same study displayed hypo-responsiveness to $a$-GalCer. ${ }^{11}$ Contrary to observations in MS, in CFS/ME patients, iNKT cells were reportedly increased in severely affected CFS/ME patients and were not altered in less affected CFS/ME patients. ${ }^{12}$

The present literature suggests that CFS/ME and MS may share some similarities in relation to reduced NK cell activity. ${ }^{5,12}$ It is likely that other cells (Tregs, iNKT, $\gamma \delta$ cells) may also be dysregulated in CFS/ME and MS. The objective of this study was to investigate peripheral regulatory cells such as Tregs, $\gamma \delta \mathrm{T}$ cells and iNKT cells in CFS/ME and MS patients compared to healthy controls and test the null hypothesis that there is no difference between these groups.

\section{Methods \\ Participant Recruitment}

Participants for this study were recruited from clinics at Griffith University and the Gold Coast University Hospital. All participants completed a questionnaire based on the Centre for Disease Control and Prevention (CDC) case definition for CFS/ME. ${ }^{1}$ MS patients had not taken any disease-modifying therapy in the 3 months prior to participation in the study. MS patients all met the 2010 revised McDonald criteria for a diagnosis of MS. ${ }^{13}$ MS disease severity was assessed using the Multiple Sclerosis Impact Scale (MSIS-29) and the expanded disability status scale (EDSS) which was undertaken by a trained neurologist. The Dr Bell's Disability Adjustment Scale was a self-reporting questionnaire completed by CFS/ME and healthy controls for fatigue scale. ${ }^{14}$ The medications used by MS and CFS/ME patients are shown in Table 2. The exclusion criteria included individuals with psychosis, epilepsy, cardiac disorders, smokers, pregnancy and breast feeding. All participants signed a Consent Form provided prior to participation. Approval for this study was obtained from the Griffith University Human Research Ethics Committee (MSC/18/13/HREC).

\section{Sample Collection}

Blood from the antecubital vein $(15 \mathrm{~mL})$ was collected into EDTA tubes for immunological assays. The blood samples were analyzed within 4 hours after collection. Further pathology screening of Full Blood Count, including white cell count, was included to eliminate abnormalities.

\section{Characterization of Tregs}

Peripheral blood mononuclear cells (PBMCs) were isolated from whole blood samples by a density gradient using Ficoll-Hypaque (GE Healthcare Life Sciences; Milan, Italy) and adjusted to a concentration of $10^{6}$ cells $/ \mu \mathrm{L}$. The combination of monoclonal antibodies (mAbs) used for Treg characterization were: CD4, CD25, CD127, CD28, CD45RA, CD152 (CTLA-4) and FoxP3. Intracellular staining of FoxP3 was performed using the $\mathrm{BD}$ Pharmingen ${ }^{\mathrm{TM}}$ transcription buffer set according to the manufacturer's instructions (BD Bioscience).

\section{Characterization of iNKT cells}

INKT cells were identified using whole blood samples. The mAbs (BD Biosciences, San Jose, CA, USA) used in determining iNKT cells were: 6B11, CD3, CD4, CD8 CD8a, CD16, CD56, CD197, CD150, CD62L, CD45RA, CD27, CD28, CD1d, PLZF, and CD45RO. Briefly, cells were stained with a combination of $\mathrm{mAb}$ for iNKT, lysed with FACS Lysing solution (BD Biosciences) and washed with PBS and fixed (BD Stabilizing Fixative) before analysis on the flow cytometer.

\section{$\gamma \delta$ T cells Assessment}

Whole blood was collected into EDTA tubes and stained with a combination of $\mathrm{mAB}$ for $\gamma \delta \mathrm{T}$ cell characterization: CD45RA, CD27, $\gamma \delta$ TCR, $\gamma \delta 1$ TCR, $\gamma \delta 2$ TCR, CD11a, CD62L and CD94. Cells were lysed with FACS Lysing solution (BD Biosciences) and washed with PBS and fixed (BD Stabilizing Fixative) before analysis on the flow cytometer. The method was previously described elsewhere. ${ }^{15}$

\section{Flow Cytometer Analysis}

The $\mathrm{mAB}$ combinations were applied to characterize the Treg, iNKT and $\gamma \delta \mathrm{T}$ cell phenotypes, receptors and transcription factors. LSR-Fortessa X-20 flow cytometer analyses (BD Biosciences) were performed using BD FACSDiva software (Version 7).

\section{Statistical Analysis}

SPSS 21 and Prism 6 were used for the statistical analysis of the data. Data from the three groups were compared using two-way ANOVA with the Dunett T3 multiple comparison test. $\mathrm{P} \leq 0.05$ was considered statistically significant. 


\section{Results}

Study participants and demographics

Participants in this study consisted of 24 CFS/ME patients (mean age $49 \pm 12.3$ years old), 11 MS patients (mean age 54 \pm 12.6 years old) and 27 healthy controls (mean age $48 \pm 12.4$ years old). The healthy controls had no incidence of fatigue

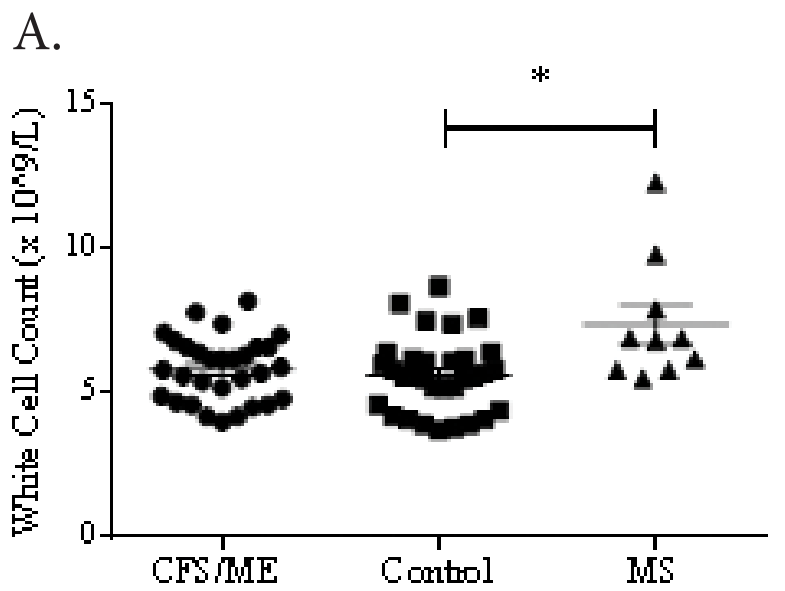

measured by the Short Form Health Survey (SF-36) (Hays et al., 1993). The MS patients in this study were comprised of relapsing remitting $(R R)(n=4)$, primary progressive (PP) $(n=2)$, secondary progressive $(S P)(n=2)$ and clinically isolated syndrome (CIS) ( $\mathrm{n}=3$ ). For MS cases, the average (SD) MSIS-29 score was 2.8 (2.9) and the mean (SD) EDSS was 2.41 (2.61).

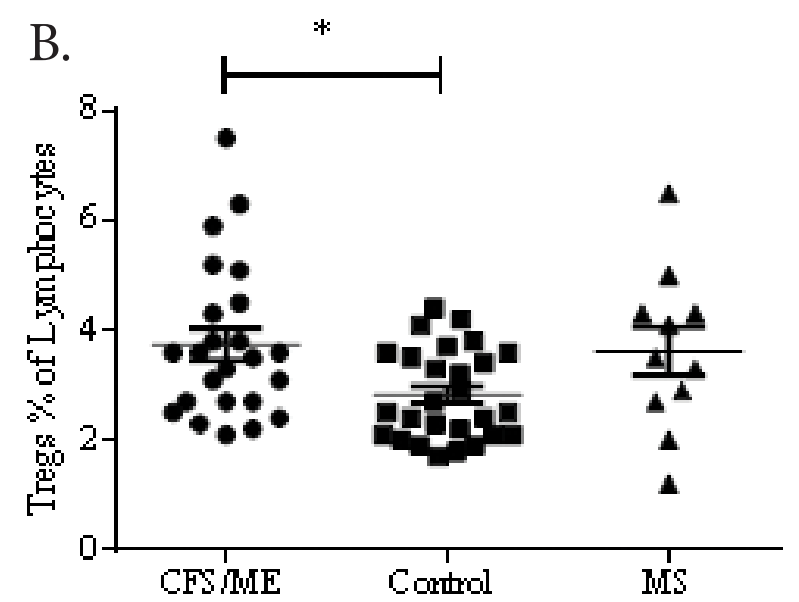

Figure 1. Total white cells and total Tregs in healthy control, CFS/ME and MS patients. (A)White Cell Count was significantly increased in the MS group compared with the healthy controls group. (B) Percentage of Treg cells were significantly increased in the CFS/ME group compared with the healthy controls group. Data is presented as mean \pm standard error of the mean. Significant difference was represented by an asterisk $\left.{ }^{*}\right), \mathrm{p} \leq 0.05$. CFS/ME is represented by the black circles (๑); Healthy controls group is represented by the black squares $(\boldsymbol{\square})$ and MS group by black triangles $(\boldsymbol{\Lambda})$.

A

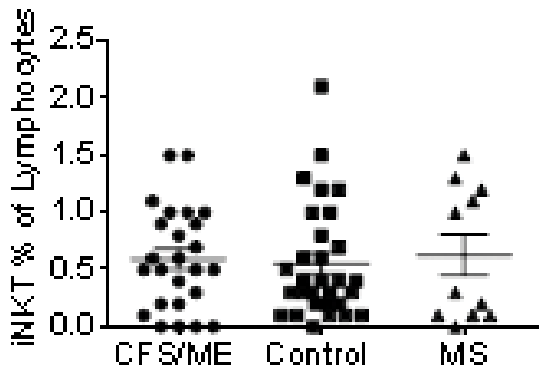

D.

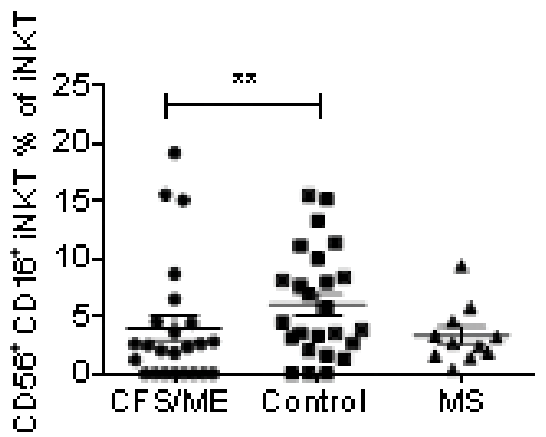

B.

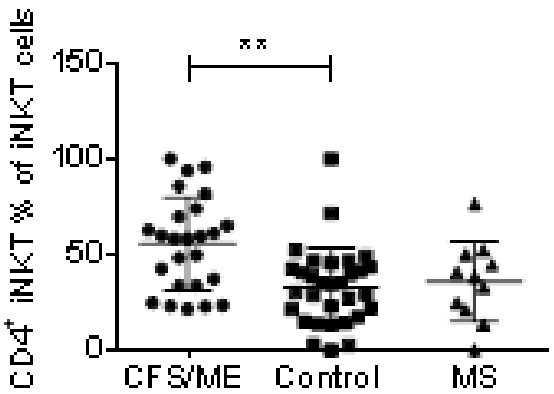

E.

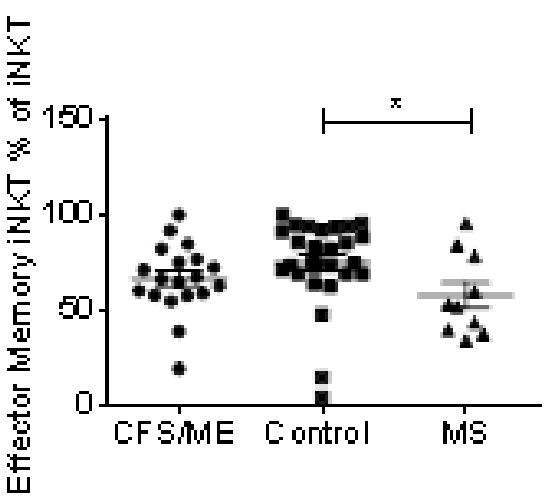

C.

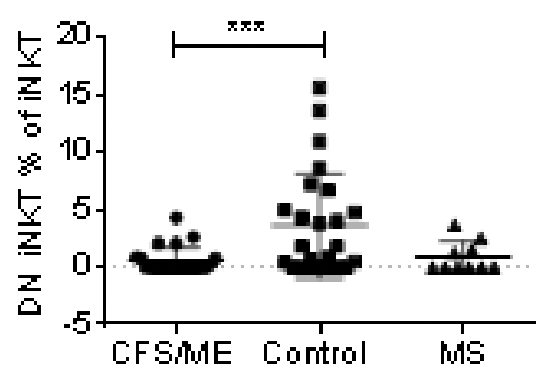

Figure 2. Total iNKT was not different amongst the three groups (3A). The percentage of CD4+iNKT cells was significantly elevated in the CFS/ME group compared with healthy controls (3B). The percentage of double negative iNKT cells (CD4-CD8 ${ }^{-}$) was significantly decreased in the CFS/ME compared with the healthy controls group (4C). The percentage of CD56 ${ }^{+} \mathrm{CD} 16^{+}$iNKT cells was significantly decreased in the CFS/ME group compared with the healthy controls group (3D). The MS group showed significant reduced percentage of effector memory iNKT cells $\left(\mathrm{CD} 45 \mathrm{RO}^{+} \mathrm{CD} 62 \mathrm{~L}-\right)$ compared with healthy controls $(3 \mathrm{E})$. Data is presented as mean \pm standard error of the mean. Significant differences were represented by asterisks $\left(^{*}\right), \mathrm{P} \leq 0.05 ;\left({ }^{*}\right), \mathrm{P} \leq 0.005$. CFS/ME is represented by the black circles $(\bullet)$; Healthy controls is represented by the black squares $(\boldsymbol{\square})$ and MS group by black triangles $(\boldsymbol{\Lambda})$. 

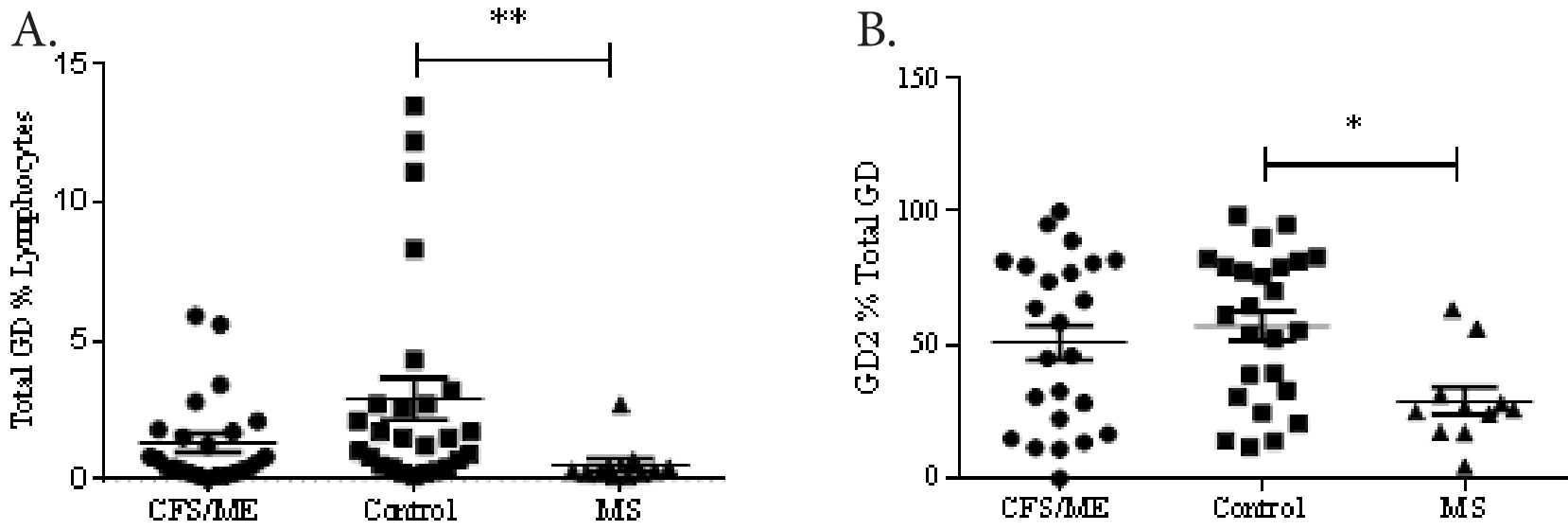

Figure 3. $\gamma \delta$ T cells and $\gamma \delta 2$ T cells in healthy controls, CFS/ME and MS patients. (A) Total $\gamma \delta$ T cells percentage of lymphocytes was significantly reduced in the MS group compared with the healthy controls group. CFS/ME group presented no difference when compared to healthy controls group. (B) $\gamma \delta 2$ T cells percentage of total $\gamma \delta$ T cells was significantly reduced in the MS group compared with the healthy controls group. CFS/ME group presented no difference compared with healthy controls group. Data is presented as mean \pm standard error of the mean. Significant differences were represented by asterisks $\left({ }^{*}\right), \mathrm{P} \leq 0.05 ;\left({ }^{*}\right), \mathrm{P} \leq 0.005$. CFS/ME is represented by the black circles $(\bullet)$; Healthy controls group is represented by the black squares $(\square)$ and MS group by black triangles $(\mathbf{\Delta})$.

For CFS/ME, the average (SD) Dr. Bell's fatigue scale score was $44.04(20)$.

\section{Immunophenotype profile in CFS/ME or MS patients}

Total white cell count was significantly increased in the MS group compared with the healthy control group but no significant differences were identified between CFS/ME and the healthy control groups (Figure 1).

The percentage of Tregs $\left(\mathrm{CD} 4^{+} \mathrm{CD} 25^{+} \mathrm{CD} 127^{\text {low }} \mathrm{FoxP} 3^{+}\right)$in lymphocytes was significantly increased in the CFS/ME group compared with the healthy controls (Figure 2).

The iNKT cells $\left(\mathrm{CD}^{+} 6 \mathrm{~B} 11^{+}\right)$percentage of lymphocytes was not different between the three groups (Figure 2A). $\mathrm{CD} 4^{+} \mathrm{iNKT}$ and $\mathrm{CD}^{+} 6^{+} \mathrm{CD} 16^{+} \mathrm{iNKT}$ cell subsets were statistically significantly increased in CFS/ME compared with the healthy control group (Figure $2 \mathrm{~B}$ and 2D, respectively), whereas the double-negative iNKT cells $\left(\mathrm{CD}^{+} 6 \mathrm{~B} 11^{+} \mathrm{CD} 4{ }^{-}\right.$ $\mathrm{CD} 8^{-}$) were significantly decreased in CFS/ME patients compared with the healthy control group (Figure 2C). MS patients demonstrated significantly decreased effector memory iNKT cells $\left(\mathrm{CD}^{+} 6 \mathrm{~B} 11^{+} \mathrm{CD} 45 \mathrm{RO}^{+} \mathrm{CD} 62 \mathrm{~L}^{-}\right)$but no significant difference in other iNKT subsets (Figure 2E). There was no significant difference in $\mathrm{CD}^{+}{ }^{+} \mathrm{iNKT}$ cells $\left(\mathrm{CD} 3^{+} 6 \mathrm{~B} 11^{+} \mathrm{CD} 8^{+}\right)$ between the three groups (data not shown). Furthermore, CTLA-4 was reduced in the MS group in comparison to the CFS/ME group, however this was not statistically significant.

Reduced total $\gamma \delta \mathrm{T}$ cells and $\gamma \delta 2 \mathrm{~T}$ cells subset percentage of lymphocytes was observed in MS patients compared with the healthy controls (Figure $\mathbf{3 A}$ and $3 \mathrm{~B}$, respectively). In the CFS/ME patients, the percentages of total $\gamma \delta \mathrm{T}$ and $\gamma \delta 2 \mathrm{~T}$ cell subsets were not altered compared with the healthy controls. There was no significant difference in the percentage $\gamma \delta 1 \mathrm{~T}$ cells subset among the three groups (data not shown).

\section{Discussion}

This study aimed to compare Treg, iNKT and $\gamma \delta \mathrm{T}$ cell subset phenotypes in CFS/ME and MS patients with the healthy controls group. A number of immunological differences were observed between CFS/ME and MS patients. The CFS/ME group had a significant increase in the $\mathrm{CD} 4^{+} \mathrm{CD} 25^{+} \mathrm{CD} 127^{\text {low }}$ $\mathrm{T}$ cells compared with the MS group. MS patients demonstrated significant reductions in the CD4-CD8-iNKT, Naïve iNKT, terminal effector memory iNKT cells percentage of Naïve iNKT, terminal effector memory iNKT cells percentage of total iNKT cells, total $\gamma \delta$ and $\gamma \delta 2$ T cells in comparison to CFS/ $\mathrm{ME}$ and healthy controls.

In this study, the MS patients were under no treatment and this group was composed of different classifications of MS (RR, SP, CIS or PP). Studies with RR-MS have previously shown to have dysfunctional suppressive function on Tregs. However, Tregs have been shown to have normal suppressive and FoxP3 expression in SP-MS patients. Treg cells functional alterations of RR-MS could be associated with a reduced expression of FoxP3 at the microRNA as well as at the protein level, as established previously. ${ }^{16}$

The significant increase in Tregs observed in CFS/ME patients may suggest sustained immunosuppression resulting from imbalances in immune homeostasis, thus mobilizing these cells to restore balance. Increases in Tregs may contribute to the significant reductions in NK activity observed in CFS/ ME. ${ }^{4}$ However, Tregs in the MS patients were similar to the healthy controls. MS patients in the stable or acute phase were reported to have steady levels of Tregs. ${ }^{17}$ Dissimilar expression of Tregs in MS and CFS/ME patients suggests different pathways of pathogenesis implicating this regulatory cell type.

CTLA-4 is a key molecule for Tregs suppressive function ${ }^{18}$ and is reduced in the MS group; CTLA-4 showed no significant difference compared with healthy controls in our study. CTLA-4 has a role in the suppression of Th2 cytokines through the induction of Th1 responses. ${ }^{19}$ Interestingly, previous data have been shown to be contradictory when examining the influence of CTLA-4 in the MS pathway. A meta-analysis research of GWAS on MS patients with emphasis on 3 loci that had previously reached genome-wide significance, resulted in 
only one of them (locus near THADA) showing differences in data. ${ }^{20}$ Others, however, have found a significant reduction in specific genes possibly affecting CTLA-4 function in MS patients. ${ }^{21}$ As previously reported in MS patients, CTLA-4 in $\mathrm{CD} 4{ }^{+} \mathrm{CD} 25^{\text {High }}$ was not altered compared with healthy controls or CFS/ME patients. ${ }^{22}$

Previous studies have shown decreased iNKT cell frequency and dysfunctional iNKT cells in MS patients. ${ }^{23}$ It is well known that iNKT cells have an essential regulatory role; nonetheless, they also act as effector cells linking the innate and adaptive immune systems. ${ }^{24}$ There was no difference in the percentage of total iNKT cells in the MS group compared with CFS/ME and healthy control groups established in this study. CD $4^{+} \mathrm{iNKT}$ cells are predominant producers of IL- 9 and IL-10 $10^{25}$ and also produce high levels of IL-4 which are responsible for Th2 differentiation. ${ }^{26}$ IL-9 has previously been described as a possible target for the inhibition of Th17 and IL-9 receptor deficiency may ameliorate experimental autoimmune encephalitis (EAE). ${ }^{27} \mathrm{An}$ increase in CD4 ${ }^{+} \mathrm{iNKT}$ cells, described in the CFS/ME group in this study, may enhance the secretion of IL-4, suggesting an increase in Th2 cells; this may act as a negative feedback loop to counteract the potential increase in Th1 cytokines. IL-10 has been reported to be increased in some CFS/ME patients, which may be in response to heightened levels of Tregs. ${ }^{28}$

Central Memory iNKT cells are memory cells and are reserves with the property of fast differentiation to effector memory cells in response to antigens. In our results, the central memory iNKT cells were not statistically different between the three groups. However, the percentage of effector memory iNKT cells was significantly reduced in the MS group compared with healthy controls. CD4CD8-iNKT or double negative iNKT cells are responsible for the balance between Th1 and Th2. The double-negative iNKT cells have been shown to be decreased in patients with autoimmune inflammatory conditions, such as systemic sclerosis. ${ }^{29}$ Many studies involving autoimmune disease have shown alterations in the Th1 and Th2 balance due to iNKT regulation. ${ }^{30,31}$ Interestingly, the percentage of CD4-CD8-iNKT cells was significantly reduced in the CFS/ME group compared with healthy controls. Besides being very similar to the MS group, the reduction of this phenotype was not significant in the MS group.

The $\mathrm{CD} 56^{+} \mathrm{CD} 16^{+}$iNKT percentage of iNKT cells was significantly reduced in the CFS/ME patients compared with the healthy controls. Remarkably, the $\mathrm{CD} 56^{+} \mathrm{CD} 16^{+}$iNKT percentage of iNKT cells in the MS group was very similar to that presented by the CFS/ME group; however, it was not significantly reduced compared with healthy controls The surface expression of $\mathrm{CD} 56^{+}$and $\mathrm{CD} 16^{+}$might suggest that this cell phenotype contains high amounts of perforin and granzyme B. The cytotoxic capacity of this cell phenotype may perhaps be closely related to natural killer cells previously shown to have reduced cytotoxic activity in the CFS/ME population. ${ }^{32}$

Total $\gamma \delta \mathrm{T}$ cells have been stated to be increased in the CNS of patients with MS, especially when compared with the frequency of those cells in the periphery. ${ }^{33}$ Previous studies in MS patients have shown effective expansion of $\gamma \delta \mathrm{T}$ cells generating high levels of IL-2, IL-10 and TNFa in response to myelin basic protein (MBP) reactive T cells vaccine. ${ }^{34} \gamma \delta$ T cells are very important inflammatory effectors that can, through the innate or adaptive immune systems, initiate a prompt response to infection. ${ }^{10}$ Nevertheless, $\gamma \delta \mathrm{T}$ cells in MS patients have previously generated contrary results as significantly reduced numbers were found in the peripheral blood compared with cerebral spinal fluid. ${ }^{35} \gamma \delta 2 \mathrm{~T}$ are the most abundant $\gamma \delta \mathrm{T}$ cells in the periphery. The reduction in the total $\gamma \delta \mathrm{T}$ and $\gamma \delta 2 \mathrm{~T}$ cells reported in this paper may suggest either apoptosis of $\gamma \delta 2$ $\mathrm{T}$ cells or translocation to the CNS, as observed in active MS cases. $^{35}$

In conclusion, $\mathrm{CFS} / \mathrm{ME}$ and $\mathrm{MS}$ are incapacitating disorders that involve the immune system and fatigue, although differently presented, can be a prominent symptom in both conditions. This paper evaluated Tregs, $\gamma \delta$ T and iNKT and their subsets in CFS/ME and MS patients. Some similarities were identified in subsets of iNKT cells in CFS/ME and MS, while other immunological changes were specific to either MS or CFS/ME patients. Importantly, the CFS/ME patients presented with a unique and altered immune profile that needs to be further explored. Tregs, $\gamma \delta \mathrm{T}$ and iNKT cells and their subsets have an important role in both disorders. However, their mechanism of action and their contribution to these conditions remains obscure. Therefore, further in-depth studies involving genetics examination on these cells in MS and CFS/ME are needed.

\section{Acknowledgments}

This research is supported by the grants from Alison Hunter Memorial Foundation, Mason Foundation (Grant Number MA43120), Queensland Government Department of Science, Information Technology, and Innovation \& the Arts Smart Futures Fund (Grant Number 216702MRE).

\section{Competing interests}

All authors declare that there is no competing interest.

\section{References}

1. Fukuda K, Straus SE, Hickie I, Sharpe MC, Dobbins JG, Komaroff A. The chronic fatigue syndrome: a comprehensive approach to its definition and study. International Chronic Fatigue Syndrome Study Group. Ann Intern Med. 1994;121:953-9.

2. Charcot J. Lectures on the diseases of the nervous system. New Sydenham Society. 1877.

3. McAlpine D, Compston, N. Some aspects of the natural history of disseminated sclerosis. The Quarterly journal of medicine. 1952;21:13567.

4. Brenu EW, van Driel ML, Staines DR, Ashton KJ, Hardcastle SL, Keane J, et al. Longitudinal investigation of natural killer cells and cytokines in chronic fatigue syndrome/myalgic encephalomyelitis. J Transl Med. 2012;10:88.

5. Brenu EW, Huth TK, Hardcastle SL, Fuller K, Kaur M, Johnston S, et al. The Role of Adaptive and Innate Immune Cells in Chronic Fatigue Syndrome/ Myalgic Encephalomyelitis. Int Immunol. 2013.

6. Curriu M, Carrillo J, Massanella M, Rigau J, Alegre J, Puig J, et al. Screening NK-, B- and T-cell phenotype and function in patients suffering from Chronic Fatigue Syndrome. J Transl Med. 2013;11:68.

7. Lim ET, Giovannoni G. Immunopathogenesis and immunotherapeutic approaches in multiple sclerosis. Expert Rev Neurother. 2005;5:379-90.

8. Maghazachi AA. Role of Natural Killer Cells in Multiple Sclerosis. International Scholarly Research Network. 2012;2012:14.

9. Viglietta V, Baecher-Allan C, Weiner HL, Hafler DA. Loss of functional suppression by CD4+CD25+ regulatory T cells in patients with multiple sclerosis. J Exp Med. 2004;199:971-9. 
10. Vantourout P, Hayday A. Six-of-the-best: unique contributions of gammadelta T cells to immunology. Nat Rev Immunol. 2013;13:88-100.

11. O'Keeffe J, Gately CM, Counihan T, Hennessy M, Leahy T, Moran $\mathrm{AP}$, et al. T-cells expressing natural killer (NK) receptors are altered in multiple sclerosis and responses to alpha-galactosylceramide are impaired. J Neurol Sci. 2008;275:22-8

12. Hardcastle SL, Brenu EW, Johnston S, Nguyen T, Huth T, Kaur M, et al. Analysis of the Relationship between Immune Dysfunction and Symptom Severity in Patients with Chronic Fatigue Syndrome/Myalgic Encephalomyelitis (CFS/ME). J Clin Cell Immunol. 2014;5:1-9.

13. Polman CH, Reingold SC, Banwell B, Clanet M, Cohen JA, Filippi M, et al. Diagnostic criteria for multiple sclerosis: 2010 revisions to the $\mathrm{McD}$ onald criteria. Ann Neurol. 2011;69:292-302.

14. Bell D. The Doctor's Guide to Chronic Fatigue Syndrome, Reading, Mass. Addison-Wesley Publishing Company, Reading, Mass1995.

15. Anane LH, Edwards KM, Burns VE, Zanten JJ, Drayson MT, Bosch JA. Phenotypic characterization of gammadelta $\mathrm{T}$ cells mobilized in response to acute psychological stress. Brain Behav Immun. 2010;24:608-14.

16. Venken K, Hellings N, Hensen K, Rummens JL, Medaer R, D’Hooghe M $\mathrm{B}$, et al. Secondary progressive in contrast to relapsing-remitting multiple sclerosis patients show a normal CD4+CD25+ regulatory T-cell function and FOXP3 expression. J Neurosci Res. 2006;83:1432-46.

17. Saresella M, Marventano I, Longhi R, Lissoni F, Trabattoni D, Mendozzi $\mathrm{L}$, et al. CD4+CD25+FOXP3+PD1-naive regulatory $\mathrm{T}$ cells in acute and stable relapsing-remitting multiple sclerosis and their modulation by therapy. Journal of Neuroimmunology. 2008;203:133-.

18. Tivol EA, Borriello F, Schweitzer AN, Lynch WP, Bluestone JA, Sharpe AH. Loss of CTLA-4 leads to massive lymphoproliferation and fatal multiorgan tissue destruction, revealing a critical negative regulatory role of CTLA-4. Immunity. 1995;3:541-7.

19. Steiner K, Moosig F, Csernok E, Selleng K, Gross WL, Fleischer B, et al. Increased expression of CTLA-4 (CD152) by T and B lymphocytes in Wegener's granulomatosis. Clin Exp Immunol. 2001;126:143-50.

20. Patsopoulos NA, Bayer Pharma MSGWG, Steering Committees of Studies Evaluating I-b, a CCRA, Consortium AN, GeneMsa, et al. Genome-wide meta-analysis identifies novel multiple sclerosis susceptibility loci. Ann Neurol. 2011;70:897-912.

21. International Multiple Sclerosis Genetics C, Hafler DA, Compston A, Sawcer S, Lander ES, Daly MJ, et al. Risk alleles for multiple sclerosis identified by a genomewide study. N Engl J Med. 2007;357:851-62.

22. Sellebjerg F, Krakauer M, Khademi M, Olsson T, Sorensen PS. FOXP3, CBLB and ITCH gene expression and cytotoxic T lymphocyte antigen 4 expression on CD4(+) CD25(high) T cells in multiple sclerosis. Clin Exp Immunol. 2012;170:149-55.
23. Hammond KJ, Kronenberg M. Natural killer T cells: natural or unnatural regulators of autoimmunity? Curr Opin Immunol. 2003;15:683-9.

24. Godfrey DI, MacDonald HR, Kronenberg M, Smyth MJ, Van Kaer L. NKT cells: what's in a name? Nat Rev Immunol. 2004;4:231-7.

25. O’Reilly V, Zeng SG, Bricard G, Atzberger A, Hogan AE, Jackson J, et al Distinct and overlapping effector functions of expanded human CD4+, CD8alpha+ and CD4-CD8alpha- invariant natural killer T cells. PLoS One. 2011;6:e28648.

26. Montoya CJ, Pollard D, Martinson J, Kumari K, Wasserfall C, Mulder $\mathrm{CB}$, et al. Characterization of human invariant natural killer T subsets in health and disease using a novel invariant natural killer T cell-clonotypic monoclonal antibody, 6B11. Immunology. 2007;122:1-14

27. Nowak EC, Weaver CT, Turner H, Begum-Haque S, Becher B, Schreiner B, et al. IL-9 as a mediator of Th17-driven inflammatory disease. J Exp Med. 2009;206:1653-60.

28. Nakamura T, Schwander SK, Donnelly R, Ortega F, Togo F, Broderick G, et al. Cytokines across the night in chronic fatigue syndrome with and without fibromyalgia. Clin Vaccine Immunol. 2010;17:582-7.

29. Sumida T, Sakamoto A, Murata H, Makino Y, Takahashi H, Yoshida $\mathrm{S}$, et al. Selective reduction of $\mathrm{T}$ cells bearing invariant $\mathrm{V}$ alpha 24J alpha Q antigen receptor in patients with systemic sclerosis. J Exp Med. 1995;182:1163-8.

30. Furlan R, Bergami A, Cantarella D, Brambilla E, Taniguchi M, Dellabona $\mathrm{P}$, et al. Activation of invariant NKT cells by alphaGalCer administration protects mice from MOG35-55-induced EAE: critical roles for administration route and IFN-gamma. Eur J Immunol. 2003;33:1830-8.

31. Pal E, Tabira T, Kawano T, Taniguchi M, Miyake S, Yamamura T. Costimulation-dependent modulation of experimental autoimmune encephalomyelitis by ligand stimulation of $\mathrm{V}$ alpha $14 \mathrm{NK} \mathrm{T}$ cells. J Immunol. 2001;166:662-8.

32. Huth TK, Brenu EW, Nguyen T, Hardcastle SL, Johnston S, Ramos S, et al. Characterization of Natural Killer Cell Phenotypes in Chronic Fatigue Syndrome/Myalgic Encephalomyelitis. J Clin Cell Immunol 2014;5:1-8.

33. Wucherpfennig KW, Newcombe J, Li H, Keddy C, Cuzner ML, Hafler DA Gamma delta T-cell receptor repertoire in acute multiple sclerosis lesions. Proc Natl Acad Sci U S A. 1992;89:4588-92.

34. Stinissen P, Zhang J, Vandevyver C, Hermans G, Raus J. Gammadelta T cell responses to activated $\mathrm{T}$ cells in multiple sclerosis patients induced by T cell vaccination. J Neuroimmunol. 1998;87:94-104.

35. Droogan AG, Crockard AD, Hawkins SA, McNeill TA. Gamma delta T cell distribution in cerebrospinal fluid and peripheral blood of patients with multiple sclerosis. J Neurol Sci. 1994;126:172-7. 